\title{
El uso de las ecuaciones como guía para pensar y resolver problemas: una aproximación a la enseñanza de la física
}

\author{
$J P$. Valcárcel $M$. \\ juval@usco.edu.co \\ Facultad de Educación \\ J. M. Perea Espitia \\ juanperea@telecom.com.co \\ Facultad de Educación.
}

\section{Resumen}

entro de las propuestas para la enseñanza de las ciencias podemos diferenciar diversos procedimientos que son eficaces si se dispone de los conocimientos adecuados, reorientando estos hacia la comprensión de los núcleos conceptuales básicos de la ciencia en particular, en la que pueden considerarse principalmente tres tipos de contenidos verbales: datos, conceptos y principios.

En la enseñanza de la Física el seguimiento de estas condiciones se aplica a la solución de problemas y, útilizando la apropiada formulación de ecuaciones, se facilita a los estudiantes la comprensión de esta ciencia.

Palabras clave: enseñanza de la Física, formulación, datos, ecuaciones.

\footnotetext{
Abstract

Among the proposals for the teaching of science, we can differentiate various procedures that are effective if they have the appropriate knowledge, reoriented towards the understanding of basic conceptual core of science in particular, in which
}

we can consider mainly three types of verbal content: data, concepts and principles.

In the teaching of physics monitoring of these conditions apply to solving problems and using the appropriate formulation of equations, students are provided with the understanding of this science.

Keywords: Physics teaching, principles, data, and equations.

\section{Introducción}

En el ejercicio de la docencia, los contenidos verbales han desempeñado un papel central en el aprendizaje. Estos contenidos verbales se refieren principalmente a datos o hechos que afirman o reconocen algo que existe. Hay muchos datos sobre todo posible existente en forma real: gases, líquidos, sólidos, etc., pero, por ejemplo, el dato "sólido" no se comprende si no se le da sentido y significado a través de un concepto. 
Datos y conceptos aún no son completos en las actividades de aprendizaje-enseñanza si no se establecen unos principios. El gradiente en esta dinámica: hechos, conceptos específicos y principios constituirá la meta para lograr una comprensión de los contenidos aún de los más generales.

Un aprendizaje significativo en la comprensión de la Física es el conocimiento y aplicación de los datos, conceptos y principios a través de la resolución de problemas utilizando una apropiada formulación de ecuaciones.

El lenguaje de la física son las matemáticas. Con el fin de estudiar física en serio, hay que aprender las matemáticas. Para iniciar un proceso de aprendizaje de la Física, a través de la resolución de problemas, utilizando una apropiada selección de ecuaciones, se sugiere seguir el siguiente esquema:

a. Leer el texto. Si el libro no le suministra claridad, buscar uno menos complejo. Es preciso tener una actitud favorable a la comprensión o deseo de aprender lo cual requiere una práctica más continuada.

b. Asistir a clase. El seguimiento presencial e interesado de la asignatura facilita el aprendizaje. Recordar que hay una gran diferencia entre la enseñanza de la escuela secundaria y la de la universidad donde los profesores están altamente especializados en su campo de estudio y hay niveles de exigencia distintos en una y otra etapa educativa.

c. Tomar notas y hacer preguntas. Trabajar con sus ejemplos y si encuentra dificultades preguntar si éstas son las matemáticas o la física, o ambas. Utilizar unos cinco minutos, inmediatamente después de clase, y repasar las notas.

d. Leer nuevamente el texto y resaltar lo principal.

e. Hacer las tareas. Trabajar con cada problema detalladamente, resolver cada ecuación, revelar y entender cada presuposición, y después intentar derivar la solución otra vez sin mirar en el libro. Al hacer esto constantemente habrá más facilidad en la resolución de los problemas.

f. Repasar, revisar y preguntar, siguiendo el diagrama de flujo mostrado en la Figura 1.

"Un gran descubrimiento soluciona un gran problema pero solo hay un pequeño

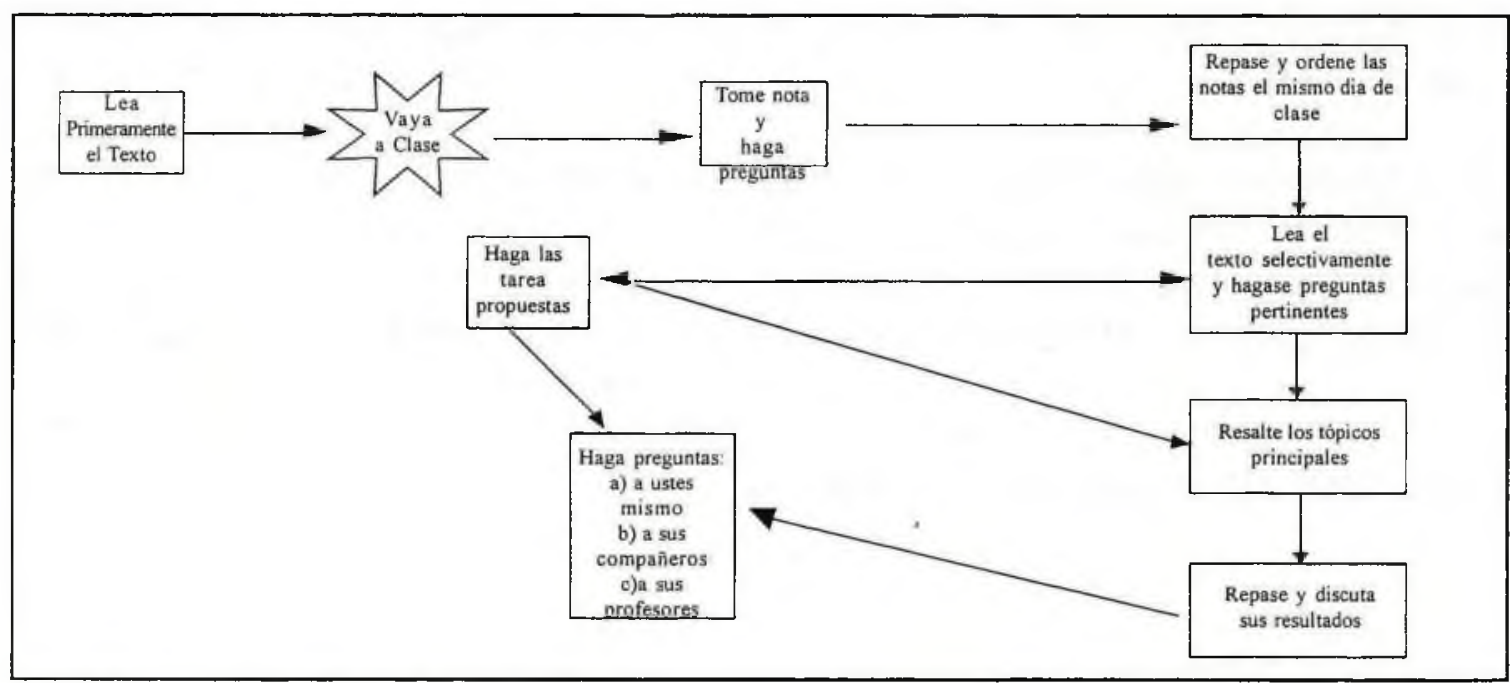

Figura 1. Diagrama de flujo que muestra los pasos necesarios para una óptima comprensión y ayuda en la solución de problemas en Física. 


\section{PaIneIaSurcolombiana 17}

descubrimiento en la solución de cualquier problema. Su problema puede ser modesto; pero si este estimula su curiosidad y le sirve a sus propiedades inventivas, y si estas las resuelve mediante sus propios medios, se experimenta la tensión y se goza del triunfo del descubrimiento. Tales experiencias en una edad susceptible puede crear un gusto especial por el trabajo mental y puede crear una satisfacción por el trabajo mental que dejan su huella en la mente y en el carácter por toda la vida" 202

\section{Cómo Aprender Física}

La diferencia entre aprender física y matemáticas es doble; primero se presenta un problema de física, después se expresa en un problema matemático, y luego se soluciona el problema, cerciorándose de que en cada paso las manipulaciones de la notación matemática corresponden a la realidad. Hay dos precisiones en la solución de un problema, el matemático y el físico.

Una forma de aprender y utilizar las exigencias de las matemáticas es desarrollar una intuición sobre el concepto físico, o por lo menos desarrollar una intuición sobre la manipulación de las matemáticas que corresponde a la realidad física. La manera de desarrollar tal intuición es considerar cómo el concepto físico se utiliza matemáticamente al solucionar problemas de la Física.

La Física aplicada comprende el conocimiento, entendimiento de los principios y leyes generales de la Física de los diferentes campos básicos. En principio la Física se divide en dos grandes grupos: visibles que se suceden a velocidades mucho más pequeñas que la velocidad de la luz $(300.000 \mathrm{~km} / \mathrm{s})$.

b. Física Moderna, que se encarga de todos aquellos fenómenos producidos a la velocidad de la luz o con valores cercanos a ella. Esto debido a que la Física Clásica no describe con precisión los fenómenos que se suceden a la velocidad de la luz.

La Tabla 1 muestra las divisiones "básicas" que integran la Física Clásica y las de la Física Moderna.

Con el fin de obtener un dominio que haga más accesible el aprendizaje, ya que en cuanto más estable es una teoría de dominio mayor es su consistencia, se enumeran a continuación varios pasos que procuran obtener el completo dominio de resolver problemas en Física:

Ejercitar las técnicas de resolución de problemas y casos prácticos de aplicación directa de los temas desarrollados en las clases teóricas.

Usar correctamente los métodos matemáticos como herramienta imprescindible.

Saber deducir la ecuación diferencial que rige el movimiento en cada tipo de vibración.

Análisis y síntesis, razonamiento crítico y resolución de problemas.

* Transmitir con claridad y rigor información, ideas, problemas y soluciones de forma oral y escrita.

a. Física Clásica, que estudia los fenómenos

${ }^{202}$ Tomado de: How to solve it, a new aspect of mathematical method, G. Polya, Princeton University Press (1973). 
FÍSICA CLÁSICA

Mecánica (fenómenos relacionados con el movimiento de los cuerpos)

Estática (cuerpos en equilibrio)

Cinemática (tipos de movimientos sin

importar las causas)

Dinámica (causas por las que los cuerpos ya

no están en equilibrio)

Termodinámica (Fenómenos térmicos)

Electromagnetismo (interacción de los campos

eléctricos y magnéticos)

Óptica (Fenómenos relacionados con la luz)

Acústica (Sonido y fenómeno de la audición)
FÍSICA MODERNA

Física Cuántica (energía formada de "cuantos")

Física de la Relatividad

Tabla 1. Disciplinas que comprenden la Física Clásica y la Física Moderna.

- Reunir e interpretar datos relevantes y emitir juicios.

- Trabajo en equipo.

" Desarrollo de habilidades de aprendizaje para emprender estudios posteriores.

- Conocimiento, gestión y uso de las tecnologías de la información y la comunicación.

Aplicar los conocimientos adquiridos a la práctica.

Un ejemplo:

\section{"El uso de las ecuaciones como guía para pensar y resolver problemas. ${ }^{203}$}

La Ciencia es el estudio de las leyes de la naturaleza. Las más básicas de ellas son las leyes de la Física, la mayoría de las cuales

${ }^{203}$ PGHewitt@aol.com muestran cómo los conceptos se conectan unos con otros. ¿Pero el estudio de estas ecuaciones mejora la comprensión del estudiante? No siempre, ya que a menudo en un curso introductorio los estudiantes están tentados (o aún motivados) para memorizar las ecuaciones o guardarlas en una lista manual y entonces, cuando las confrontan con un problema para mirar cuál de ellas es la relevante pueden sustituirla por números.

Se obtiene poco entendimiento de estos resultados. O peor, las ecuaciones pasan a un segundo plano en un curso introductorio y se les da poca o ninguna atención (como sucede con algunos libros populares de Física que presumen no tener ecuaciones).

Las ecuaciones en cursos más avanzados toman un lugar de preferencia con el fin de mostrar, en una forma concisa, las relaciones que existen en la naturaleza y pueden ser manipuladas por los estudiantes para conocer conexiones nuevas y obtener inadvertidos 


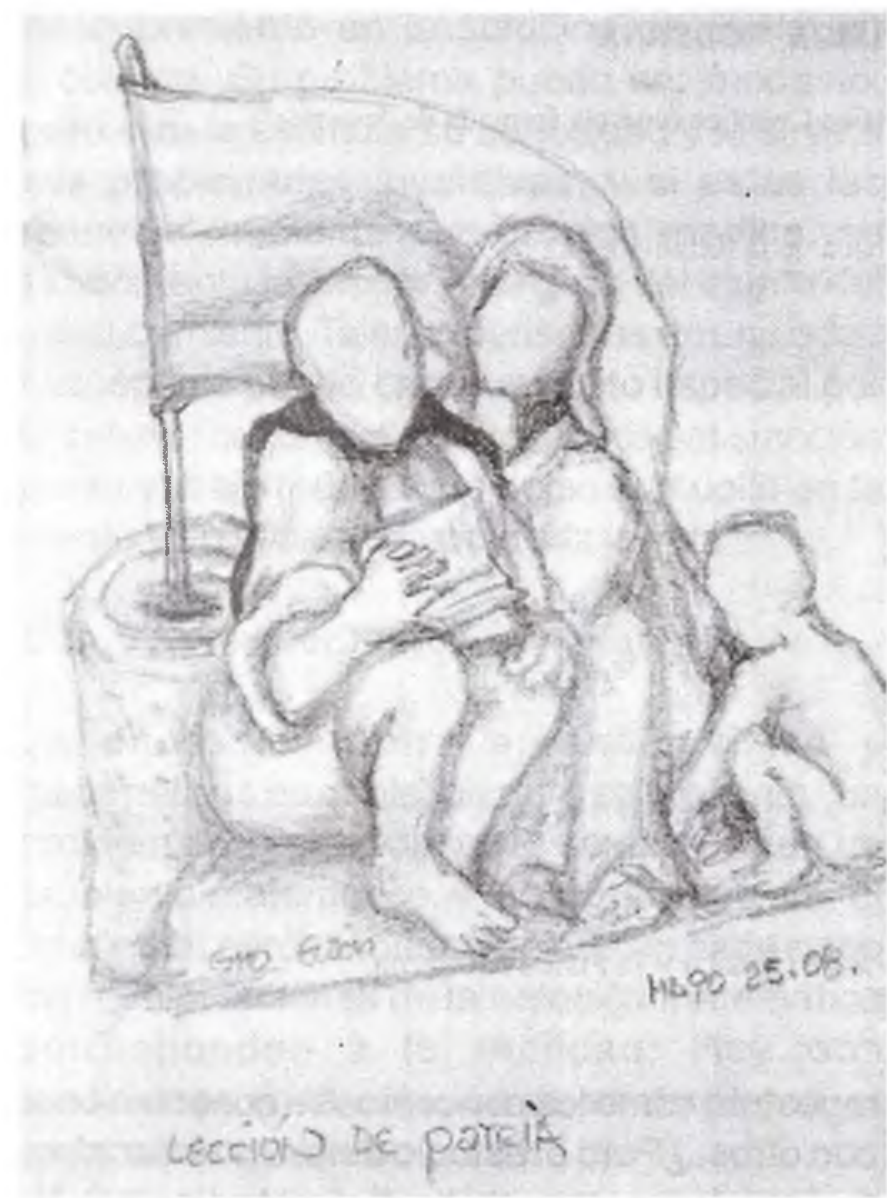

resultados. ¿La mayoría de este enfoque sobre las ecuaciones se aplica solamente en cursos avanzados? La experiencia es que el énfasis en las ecuaciones no solo es posible en cursos introductorios, sino que es altamente necesario.

La enseñanza de las ecuaciones como guias para pensar es similar a la ilustración de las partituras musicales para guiar a los músicos. ¿Qué significado deben tener las ecuaciones de la Física?

\section{Símbolos}

Los símbolos representan conceptos.

El significado de los símbolos ha sido el foco conceptual en los cursos de Física durante muchos años. El énfasis va más allá de lo que los símbolos representan, sino lo que ellos significan- cómo su presencia en las ecuaciones muestra las conexiones en la naturaleza. El centro de atención en estas conexiones puede hacer de un curso introductorio más agradable al ser más significativo y más eficaz.

Existen dos aspectos en la idea de tomar las ecuaciones como guía para pensar. Uno es justamente el examen y apreciación de las ecuaciones y lo que revelan respecto a las relaciones entre los conceptos (la dependencia de la raíz cuadrada inversa de las fuerzas eléctricas y gravitacionales sobre la distancia, por ejemplo, o la proporcionalidad del impulso al cambio de momentum).

Cada ecuación es capaz de sostenerse, de darse la vuelta, de examinarse y discutirse de la misma forma que se hace con una pintura - una composición musical para los estudiantes de arte o de música.

El segundo aspecto es la manipulación y combinación de las ecuaciones en búsqueda de un resultado particular, antes que los números, sean insertados. Después que se haya discutido la independencia del movimiento horizontal y vertical de proyectiles (jun intrigante descubrimiento para los recién llegados!), se considera el siguiente problema: Una bola de tenis que se mueve horizontalmente supera aproximadamente la valla y aterriza en el campo de tenis.

(a) Dado que la bola supera la valla a una altura y, la distancia de la base de la malla al borde de la cancha es $x$ muestre que la máxima velocidad de la bola es

$$
\frac{x}{\sqrt{\frac{2 y}{g}}}=\left(\text { o equivalentemente, } \sqrt{\frac{x^{2} g}{2 y}}\right)
$$

Una vez que los estudiantes lleguen a familiarizarse con las ecuaciones de la cinemática y su simbología, este problema no resulta difícil. ¿Qué pasa con los números, los 
cuales también son parte de la Física? Solamente después de la solución simbólica, se hace la pregunta (b):

Dado que la altura de la bola cuando pasa sobre la malla es $0,92 \mathrm{~m}$ y la distancia al borde de la cancha es $11,9 \mathrm{~m}$, encuentre la máxima velocidad de la bola. Y tal vez haya una pregunta (c):

¿Qué tanto difiere su respuesta si la malla fuera levemente más alta?

Los estudiantes que no han aprendido vía uso de los símbolos estarán intimidados por el problema simbólico propuesto y prefieren mayormente considerar la parte (b), con nada de la (a). Para estos estudiantes, ensayo y error es el modo preferido. Y la parte (c) les parece una pregunta "truculenta". ¿No son estos los estudiantes quienes escogen no continuar en Física?

Por otra parte, los estudiantes que hayan aprendido a pensar en el significado de las notaciones en Fisica y hayan examinado las ecuaciones por lo que ellas significan es una bienvenida en la solución de problemas simbólicos. Las condiciones donde los símbolos se cancelan y ahorran pasos en una solución son a menudo automáticas en problemas simbólicos. Mucho más importante es tener en cuenta:
Cuando los problemas se expresan en símbolos, y los números se dejan para más tarde la tarea del estudiante es pensar ya que las calculadoras no lo hacen. Los estudiantes piensan conceptos. ¿No es entonces la enseñanza hecha sobre símbolos y sus significados en un curso introductorio un esfuerzo que vale la pena?"

Bibliografía

G. Polya, How to solve it, a new aspect of mathematical method, Princeton University Press (1973).

Paul G. Hewitt, Equations as Guides to Thinking and Problem Solving, City College of San Francisco, San Francisco, CA

http://ocw.upm.es/ingenieria-agroforestal/fisicaaplicada-a-la-ingenieria

http://es.scribd.com/doc/5506716/Division-dela-fisica

http://fsc729.ifreepages.com/ aprenderfisica.html 


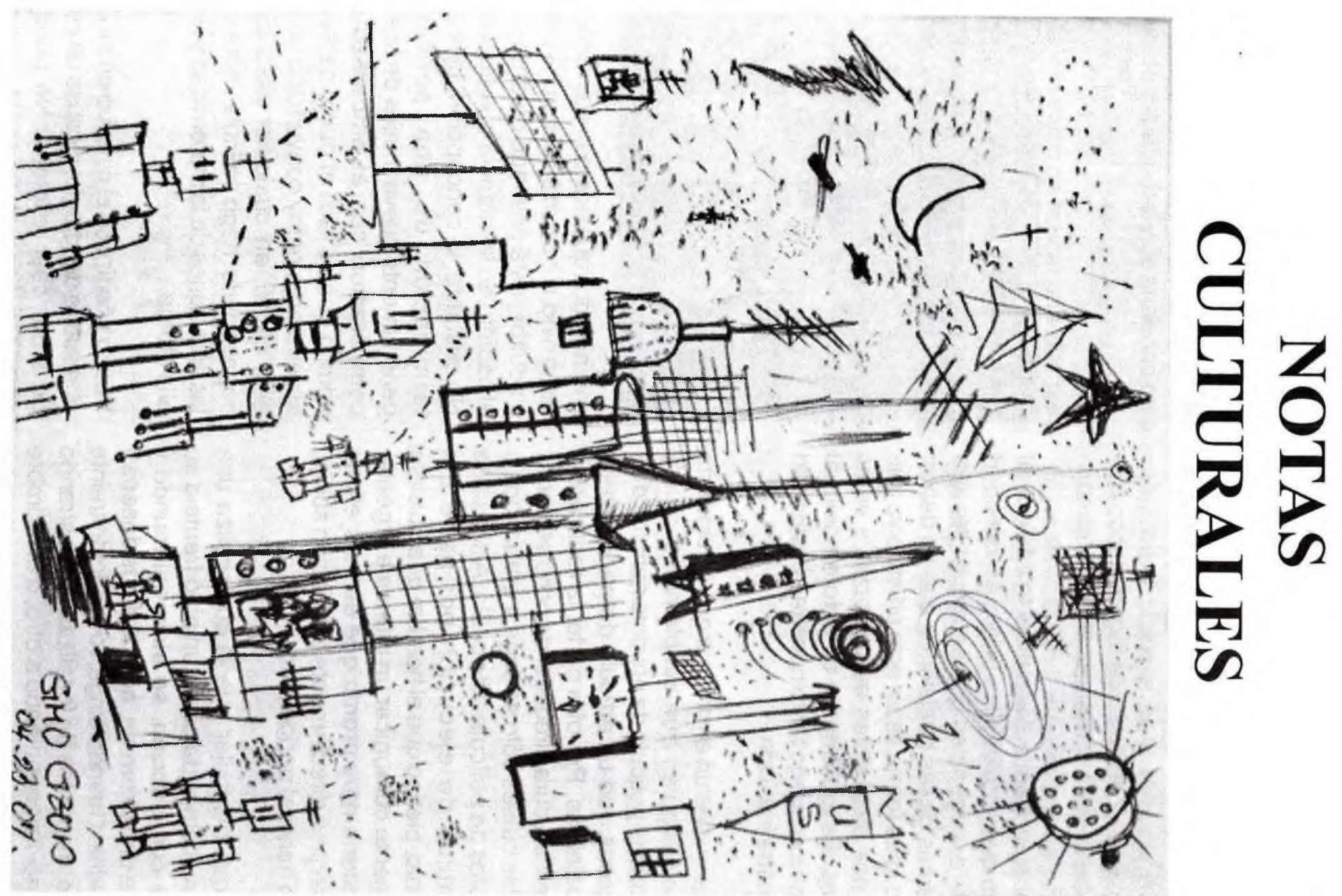

